\title{
Estimación del peligro sísmico en el centro de Baja California: Un modelo consistente entre las regiones del Golfo de California y la Peninsular
}

\author{
Roberto Ortega ${ }^{1, *}$, Dana Carciumaru², Luis Quintanar ${ }^{3}$, Reynaldo Rubio ${ }^{4,5}$ \\ ${ }^{1}$ CISESE, Miraflores 334, Col. Bellavista, La Paz, BCS, 23500. \\ ${ }^{2}$ Orbis Consultores en Geología y Geofísica, Retorno Calafia 120, Col. Calafia, La Paz, BCS, 23054 \\ ${ }^{3}$ Instituto de Geofísica UNAM, Ciudad Universitaria, Coyoacán 04510, México D.F. \\ ${ }^{4}$ Geobaja Ingeniería. Márquez de León 343, Centro, La Paz, BCS 23000. \\ ${ }^{5}$ Instituto Tecnológico de La Paz. Boulevard Forjadores 4720, 8 de Octubre, La Paz, 23080. \\ *ortega@cicese.mx
}

\section{Resumen}

En este trabajo se presenta un estudio probabilístico de peligro sísmico en la parte central de la Península de Baja California, la cual comprende principalmente la región de la Reserva de la Biosfera del Vizcaíno. La zona ha sido poco estudiada debido a su baja densidad de población; sin embargo, en esta parte de la península se han registrado las mayores aceleraciones de Baja California Sur de los últimos 15 años. Se observa que en esta región las fallas transformantes son de mayor importancia para periodos de recurrencia cortos (<1000 años), mientras que las fallas peninsulares controlan el peligro para grandes periodos de retorno ( $>1000$ años). Los resultados muestran que es importante conocer la localización de las fallas activas especialmente en el valle del Vizcaíno, donde los espesores sedimentarios cubren su localización. Se propone escoger periodos de recurrencia a partir de la información geológica. Además, se describe la dificultad de conciliar los modelos Gutenberg-Richter y Característicos, debido a que el modelo Característico no puede ser expresado con una distribución de Poisson para periodos típicos de los mapas de peligro sísmico en esta zona, porque en general las fallas cuaternarias tienen recurrencias de decenas de miles de años, mientras que la información contenida en los mapas de peligro sísmico se refieren a pocos cientos de años. La alternativa que se presenta es simple y se recomienda para la toma de decisiones en la construcción de estructuras esenciales (Tipo A). Esta alternativa recomienda usar como periodo de retorno del mapa aquel que corresponda al del ciclo del modelo de la falla característica más cercana al sitio. De otra forma los mapas de peligro sísmico se encuentran sub-valuados a pesar de que se apliquen factores de seguridad en el diseño.

Palabras clave: peligro sísmico, Baja California Sur, sismo característico, distribución de Poisson.

\begin{abstract}
We present results of a probability seismic hazard analysis in the central part of the Baja California Peninsula, which comprises mainly the Vizcaino Biosphere Reserve. This part of the peninsula has been poorly studied because it has the lowest population density of the region. However, the highest acceleration values in Baja California Sur, Mexico, during the last 15 years have been recorded in this area. It is observed that transform faults control the seismic hazard for short recurrence periods $(<1000$ years), whereas the peninsular faults are of major importance for long recurrence periods (> 1000 years). Moreover, it is necessary to have a detailed map of the seismic sources, especially those from the Vizcaino valley, where sedimentary fill covers the geological structures. We propose choosing return periods that are consistent with the observed geological fault. In addition, we discuss the difficulty to conciliate the Gutenberg-Richter and Characteristic models. The Poisson distribution is not adequate for the Characteristic model in a Probabilistic Seismic Hazard Map (PSHM) because they span different time lapses; while the PSHM model is commonly used for intervals of a few hundred years, the Characteristic model for Quaternary faults is for several tens of thousands years. A simple solution for hazard
\end{abstract}


maps, especially for essential facilities, is to choose a return period that corresponds to the characteristic fault that is nearest to the site. Otherwise the map will underestimate the seismic hazard even though design correction factors are applied.

Keywords: seismic hazard, Baja California Sur, Characteristic Earthquake, Poisson distribution.

\section{Antecedentes}

El presente trabajo muestra los resultados de un esfuerzo de 10 años para analizar el peligro sísmico en Baja California Sur. Es necesario enfatizar que el término Peligro Sísmico utilizado a lo largo de todo el artículo es en el sentido del término Seismic Hazard en inglés. Claramente, este tipo de estudios requiere tomar en cuenta los trabajos previos, en específico, este artículo está basado en varios reportes de estimación de peligro sísmico para México (Tanner y Sheppard, 1997). En Baja California Sur se ha analizado el peligro sísmico a la luz de fallas Cuaternarias, pero solamente se ha estudiado la parte sur. Sin embargo, la parte central también tiene alto potencial de amenaza debido a que se han registrado aceleraciones de 600 gales en sitios donde no existían fallas conocidas (Munguía, 2014, comunicación personal). En esta región la densidad de población es baja y no se tienen redes sísmicas densas; sin embargo, parte de la actividad sísmica ha sido registrada por estaciones regionales, o por campañas temporales de registro para análisis de réplicas o enjambres. Además, esta zona presenta alto potencial de desarrollo debido a la actividad minera y turística, por lo que es muy importante realizar estudios técnicos a fin de mitigar los efectos de desastres naturales, especialmente de peligro sísmico. En este artículo se describe primero detalladamente el ambiente tectónico, después la metodología para la estimación de peligro sísmico utilizando los modelos de Gutenberg-Richter y Característico, y finalmente se presentan los resultados más importantes que describen la sensibilidad de los cálculos, utilizando como ejemplo un sitio en Bahía Asunción, el cual ha registrado la aceleración máxima reportada en el estado de Baja California Sur en los últimos 15 años.

\section{Ambiente tectónico}

El Golfo de California es un proto-océano que se encuentra bajo un proceso permanente de extensión continental transtensional activa, a lo largo del límite de placas oblicuas divergentes. La deformación en esta región está regida por los sistemas de fallas normales y de rumbo (Angelier et al., 1981; Fletcher y Munguía, 2000; Umhoefer et al., 2002; Plattner et al., 2007). El eje del Golfo de California está marcado por fallas de rumbo de desplazamiento lateral derecho separadas por centros cortos de expansión. En el sureste de la península de Baja California se encuentra un conjunto de fallas normales con rumbo norte buzando principalmente al este, conocidas como sistema del margen del golfo (Fletcher y Munguía, 2000).

El inicio de la expansión del fondo marino en el sur del Golfo de California ocurrió hace 6-2.4 Ma (Lizarralde et al., 2007; Umhoefer et al., 2008). La expansión no fue acompañada por el cese del rifting continental, ni con una definición completa del límite de la placa PacíficoNorteamericana a través del eje del golfo (DeMets, 1995; Fletcher y Munguía, 2000; Plattner et al., 2007; Lizarralde et al., 2007). Esta región, por lo tanto, ofrece la oportunidad de estudiar el desarrollo del sistema de fallas en un momento crítico durante la transición de estado de rift a drift cuando la expansión oceánica ha comenzado mientras el rifting continental sigue activo. La tasa de expansión a través de la dorsal de Alarcón en la parte sur del Golfo de California es más lenta que la del límite de la placa Pacífico Norteamericana ( $90 \%$ del total), indicando que además de las fallas transformes y los centros de expansión del golfo, las estructuras también están acomodando la deformación en esta región (DeMets, 1995; Fletcher y Munguía, 2000; Plattner et al., 2007). La sismicidad (Munguía et al., 2006) y las relaciones geomorfológicas (Fletcher y Munguía, 2000; Busch et al., 2006, 2007; Maloney et al., 2007) indican que el conjunto de fallas normales del extremo sur de la Baja California peninsular está activo y actúa como una zona de cizalla débil que contribuye a trasladar los bloques de la península de Baja California fuera de la parte continental de México (Plattner et al., 2007). La margen del golfo es una zona de extensión, de disminución de la elevación y de adelgazamiento de la corteza (Lizarralde et al., 2007). Mientras el conjunto de fallas del golfo proporciona una contribución relativamente menor a la divergencia de placas de esta región, estas fallas normales controlan la topografía de la parte extrema sur de la península de Baja California y producen terremotos moderados (Fletcher y Munguía, 2000). Las fallas delimitan cuencas cuaternarias y desplazan depósitos aluviales cuaternarios (Fletcher y Munguía, 2000; Busch et al., 2006, 2007; Maloney et al., 2007).

Durante la mayor parte del Cenozoico la parte oeste de América del Norte, a la latitud de Baja California, sufrió la subducción de la placa Farallón (Stock y Hodges, 1989). Mientras la placa Farallón continuaba subducida (Figura 1a), la placa del Pacífico localizada al oeste de Farallón entraba en contacto con América del Norte y empezaba el proceso de subducción bajo la placa Norteamericana, iniciando el desarrollo del punto triple de Mendocino y Rivera a lo largo del sur de California y del norte de Baja 
California (Atwater, 1970; Stock y Hodges, 1989). Mientras la subducción de la placa del Pacífico continuaba, el punto triple Mendocino se movía hacia el norte y el punto triple Rivera se movía hacia el sur alargando el límite de la falla transformante lateral derecha desarrollada entre la placa del Pacífico y la Nortamericana (Stock y Hodges, 1989). Este sistema lateral derecho inició las primeras etapas del sistema de fallas de San Andrés (Atwater, 1970). Entre 20 y 12 Ma una serie de microplacas se formó a lo largo de Baja California. Las microplacas fueron capturadas por la Placa del Pacífico y la trinchera se desarrolló en una zona de falla de rumbo lateral derecha, conocida como la falla Tosco-Abreojos (Stock y Hodges, 1989; Stock y Lee, 1994). Entre 12 y 6 Ma fallas normales con rumbo NNW y $\mathrm{N}$ se desarrollaron al este de la falla Tosco-Abreojos (Figura 1b) adyacente al oeste de la región del actual Golfo de California (Stock y Hodges, 1989; Hausback, 1984; Umhoefer et al., 2002). El sistema de fallas normales que domina el margen del golfo fue parte de la repartición de la deformación entre lo que será la región del Golfo de California y la falla de rumbo Tosco-Abreojos al oeste (Stock y Hodges, 1989), o el sistema del margen del golfo fue parte de un sistema complejo de fallas transtensionales desde $12 \mathrm{Ma}$ (Fletcher et al., 2003, 2007). El régimen de divergencia oblicuo empezó aproximadamente hace 6 $\mathrm{Ma}$, cuando fallas transformantes separadas en pequeñas cuencas de rift, empezaron a formarse en el actual Golfo de California (Figura 1c) y la actividad empezó a disminuir a lo largo del sistema Tosco-Abreojos (Fletcher y Munguía, 2000; Oskin et al., 2001). Alrededor de 3.6-2.4 Ma, nueva corteza oceánica se formó a lo largo de la dorsal de Alarcón, la cual se encuentra en la región meridional de expansión del Golfo de California (DeMets, 1995; Umhoefer et al., 2008). Actualmente, el Golfo de California continúa como una región de rifting continental divergente oblicuo (Fletcher y Munguía, 2000; Umhoefer et al., 2002; Mayer y Vincent, 1999; DeMets, 1995).

\section{Fuentes sísmicas}

Las fuentes sísmicas en esta zona tienen dos orígenes: el primero corresponde a las fallas activas de la Región del Golfo de California (RGC), fallas que están asociadas al
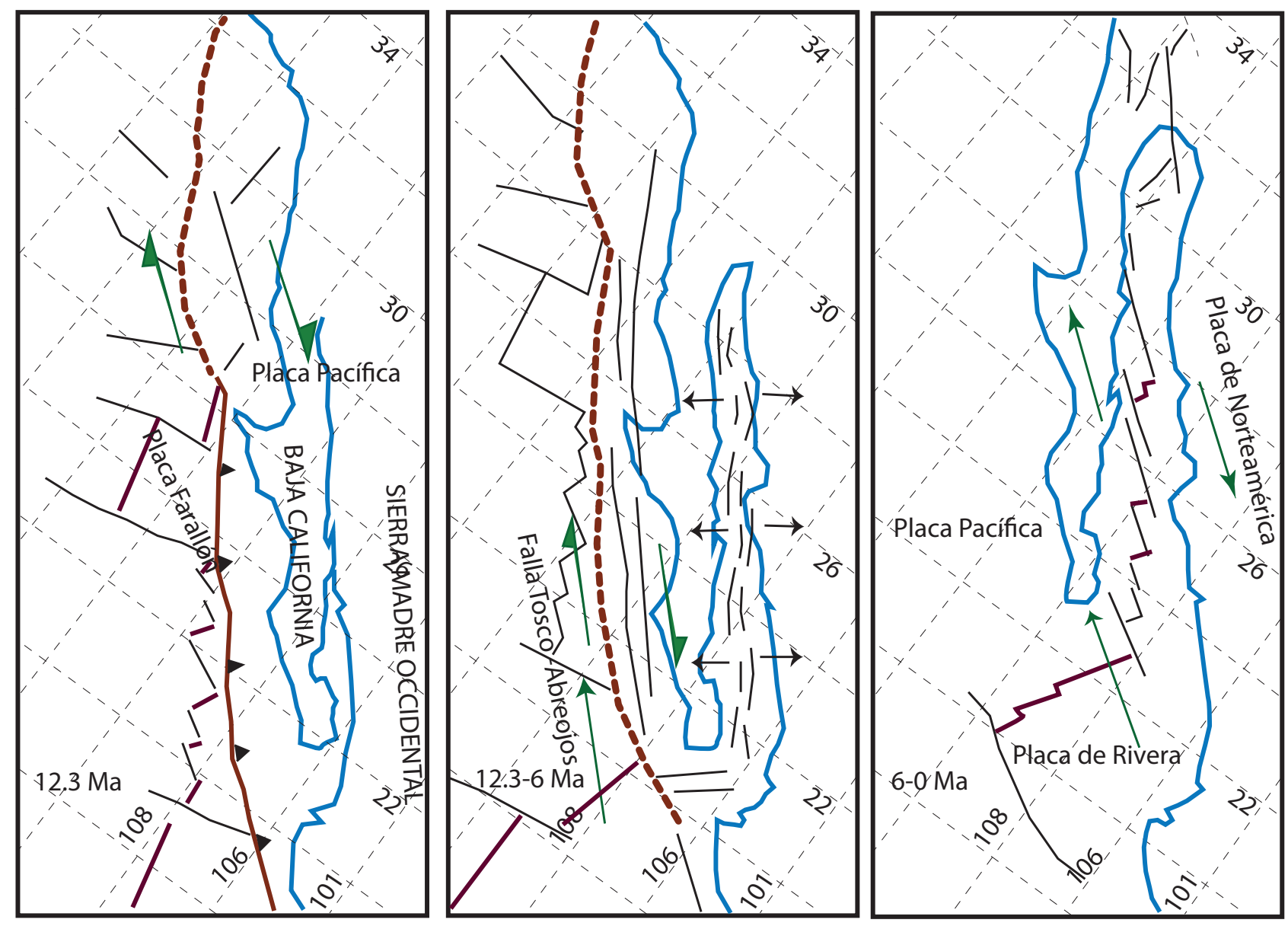

Figura 1. Historia tectónica de la región. a) Esquematización de la última etapa de la subducción de la placa de Farallón, b) Formación de la falla Tosco Abreojos, c) Inicio del régimen de divergencia oblicuo. (Modificado de Fletcher et al., 2007) 
límite entre la placa del Pacífico y la placa de Norteamérica. Esta sismicidad presenta valores de $a$ y $b$ bien establecidos de acuerdo a la ecuación de Gutenberg-Richter

$$
\log N=a-b M
$$

donde $a$ es el nivel de actividad y $N$ es el número de eventos con magnitudes mayores o iguales a $M$. El segundo tipo de fuentes se localiza en la Región Peninsular Continental (RP), asociada a las fallas activas que fueron analizadas con información geológica de la tasa de desplazamiento.

Para la RGC se considera el peligro de los terremotos con magnitudes de momento $M_{W}$ hasta 7.9. En el análisis, se consideró un valor mínimo de $M_{S}=4.5$, debido a que es el umbral de la magnitud en la cual el catálogo del Servicio Sismológico Nacional se considera completo. Aquí a es el nivel de actividad en la ecuación de Gutenberg-Richter, $\log N=a-b M$, donde $N$ es el número de eventos con magnitudes mayores o iguales a $M$. Se utilizaron varios catálogos incluyendo el del Servicio Sismológico Nacional a partir de 1974 y el catálogo del CICESE para la región de Baja California Sur. Para evaluar la integridad del catálogo se revisó el número cumulativo de eventos contra el tiempo para los eventos de diferentes regiones, utilizando los métodos de Bender (1983) y Weichert (1980).

Para la RP se utilizó el modelo de terremotos característicos, es decir, sismos que tienen un rango de magnitud angosto, en lugar de utilizar una amplia gama de magnitudes basadas en la relación de repetición de Gutenberg-Richter. Estos sismos corresponden a las fallas activas dentro de la corteza continental en la Península de Baja California. Las fallas se analizaron con datos geológicos de la tasa de desplazamiento anual y se procedió a estimar los parámetros de razón de momento sísmico, y suma de momento total para integrar el valor de recurrencia del sismo característico. Se añadieron las fallas activas que se han cartografiado por diversos autores. Este modelo es el más sensible para Baja California Sur debido a que durante los últimos 20 años de instrumentación de la península, se ha podido observar que la sismicidad local intraplaca afecta sensiblemente las aceleraciones máximas del suelo, mientras que los sismos provenientes del sistema de fallas transformantes de la Provincia Extensional del Golfo de California se rige por alta atenuación sísmica (Ortega y González, 2007; Ortega y Quintanar, 2010; Ortega y Quintanar, 2011). El análisis de las fallas activas está basado en la información geológica reportada por el Servicio Geológico Mexicano. Finalmente, para hacer un solo mapa probabilístico, las probabilidades de superación de los modelos se suman y se ponderan después de cada cálculo.

\section{Cálculo probabilístico del peligro sísmico}

A continuación se muestra el marco teórico que rige el cómputo probabilístico de peligro sísmico para las dos regiones de estudio ( $\mathrm{RGC}$ y $\mathrm{RP}$ ).
La tasa anual $\lambda\left(u>u_{0}\right)$ de que se supere el movimiento de tierra $u_{0}$ a un sitio específico se determina a partir de una suma de la distancia y la magnitud:

$$
\lambda\left(u>u_{0}\right)=\sum_{k} \sum_{l} 10^{\left[\log \left(\frac{N_{k}}{T}\right)-b\left(M_{l}-M_{r e f}\right)\right]} P\left(u>u_{0} \mid D_{k} M_{l}\right)
$$

donde $k$ es el índice del intervalo de la distancia; $l$ es el índice del intervalo de magnitud y $\mathrm{T}$ el tiempo en años del catálogo de terremotos utilizado para determinar $N_{k}$. El primer factor de la suma es la tasa anual de terremotos en una distancia de intervalo $\mathrm{k}$ y la magnitud de intervalo $l$. El valor $b$ se toma, para ser uniforme, en la mayor parte de la zona. $P(u$ $\left.>u_{0} \mid D_{k} M_{j}\right)$ es la probabilidad de que u en el sitio excederá $u_{0}$, por un terremoto a la distancia $D_{k}$ con magnitud $M_{l}$. Esta probabilidad depende de la relación de atenuación y la norma de desviación estándar del movimiento del suelo por una distancia específica y magnitud.

Nuestra hipótesis es que los sismos siguen una distribución de Poisson, es decir, con la probabilidad independiente del tiempo. La probabilidad anual que supera $u_{0}$ es igual a la tasa anual de excedencia $\lambda\left(u>u_{0}\right)$ multiplicada por las probabilidades anuales de excedencia. Después de que $l\left(u>u_{0}\right)$ se calcula para varios valores de $u_{0}$, el movimiento del suelo con una cierta probabilidad de excedencia se determina por interpolación.

A partir de:

$\dot{M} o=\mu \dot{u} L W=\sum_{M \min }^{M c} N(M) M_{O}=\sum_{M \min }^{M c} 10^{a-b m} 10^{1.5 M+16.5}$

donde: $\mu$, es el módulo de corte; $N(M)$, es el número anual de eventos en un rango de la magnitud de $M-0,05$ a 0,05 $M$; $L$, es la longitud de la falla y $W$ es el ancho de la falla, se puede obtener el valor $a$. Es decir, la ecuación (2) se reorganiza para determinar el valor a para cada falla. La ecuación (2) es una versión discreta de ecuaciones similares a las descritas por Anderson (1979).

El procedimiento para el cálculo del peligro usando el modelo de Gutenberg-Richter implica realizar el siguiente procedimiento iterativo en el rango de magnitudes entre 4.5 a 7.2, con incrementos de magnitud de 0.5 :

a) Para cada magnitud se calcula una longitud de ruptura utilizando la relación de Wells y Coopersmith (1994).

b) A continuación, una zona de ruptura de longitud calculada en (a), se flota a lo largo de la traza de la falla. c) Para cada sitio, encontramos la distancia apropiada a las rupturas flotantes y calculamos la frecuencia de excedencia (FE).

d) Se realiza una sumatoria de las FE's así calculadas para todas las zonas de ruptura flotantes.

e) Se incrementa la magnitud en 0.5 y se vuelve al paso (a).

Por supuesto normalizamos la tasa de ocurrencia de las zonas de ruptura flotantes para mantener la tasa global adecuada. 
Ahora bien, para el cálculo de RP suponemos que la falla sólo genera terremotos que rompen a lo largo de toda su longitud. Según Wesnousky (1986) se encuentra una tasa de retorno para el evento característico de momento $M_{O C}$ :

$$
\text { periodo de retorno }=\mu \dot{u} L \dot{W} / M_{O C}
$$

Comparando la ecuación (3) con la (2) se observa que se han hecho mutuamente consistentes los parámetros de sismicidad de los modelos Gutenberg-Richter y de sismo característico. El espesor de la falla se determina suponiendo una profundidad sismogénica, de $15 \mathrm{~km}$ proyectándola según su buzamiento, de manera que la anchura es igual a 15 kilómetros dividido por el seno del buzamiento. La longitud de falla se calcula a partir de la longitud total de la traza de la falla digitalizada.

Los valores de parámetros de falla se resumen en la Tabla 1.

El cálculo total de la probabilidad de peligro sísmico se basó en el árbol de ponderación para análisis de peligro sísmico de la Figura 2. Como se observa en la parte inferior de las ramas, se añadió el riesgo de las fallas y se determinó la magnitud característica a partir de la longitud máxima usando las relaciones de Wells y Coppersmith (1994), escogiendo las más apropiadas para ese tipo de tectónica.

\section{Análisis Cartográfico y Ponderación de Peligro Sísmico}

A continuación se presentan los resultados de las dos regiones que comprenden la zona en estudio: RGC y RP. Hay que destacar que es necesario realizar los cálculos de atenuación regional, magnitud máxima y periodos de retorno, los cuales se integran en el cálculo total. El cálculo de peligro sísmico se realizó limitando la región de estudio en el rango de $26^{\circ}$ a $28^{\circ}$ Lat. $\mathrm{N}$, y de $-115.5^{\circ}$ a $-111^{\circ}$ Long. W. Además se utilizaron las curvas de atenuación específicas de esta región. La RGC y RP están ponderadas con un peso de 1.0 en el cálculo total del peligro sísmico con base en la tabla lógica de la Figura 2.

Primero se llevó a cabo con una revisión cuidadosa de todas las estructuras de la región. Las fallas cartografiadas se digitizaron en un sistema de información geográfica y se cotejaron con fotos satelitales. Las estructuras activas se revisaron comparando la sismicidad local con la tendencia de las estructuras. Además se relocalizaron todos los eventos sísmicos regionales dentro de la península, a fin de tener un mapa de fallas activas (Figura 3).

Tabla 1. Valores de desplazamiento de las principales fallas.

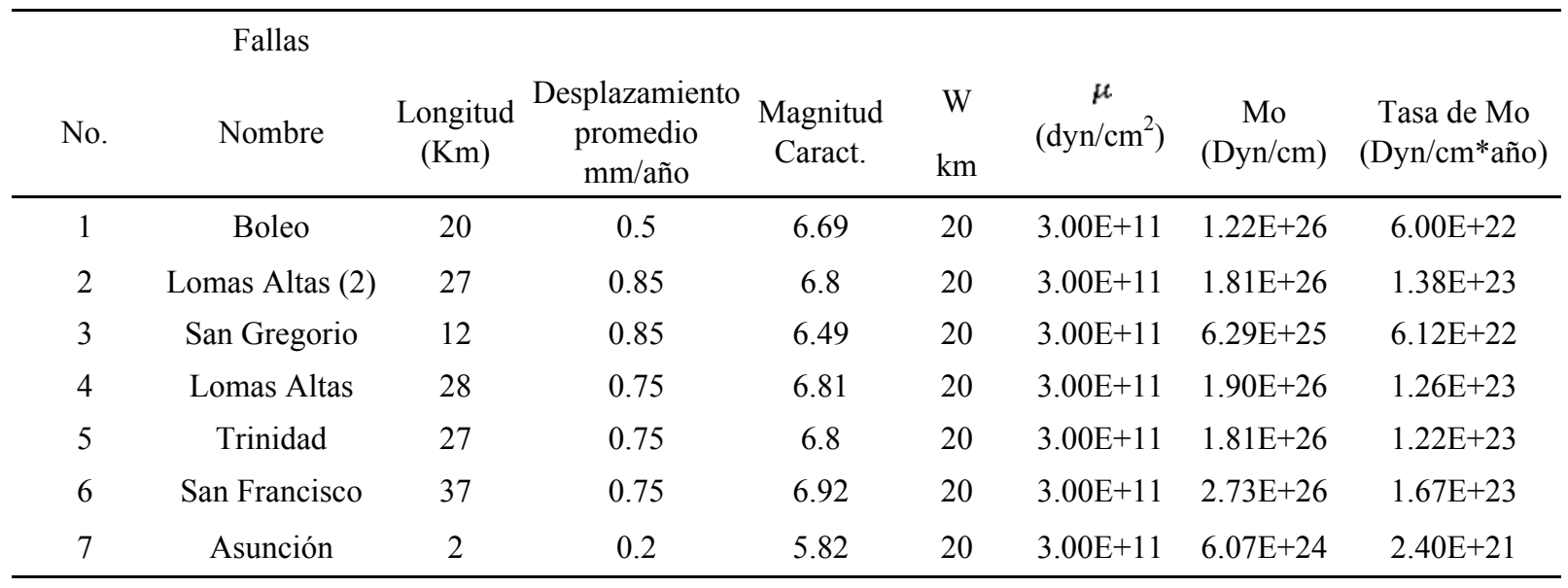

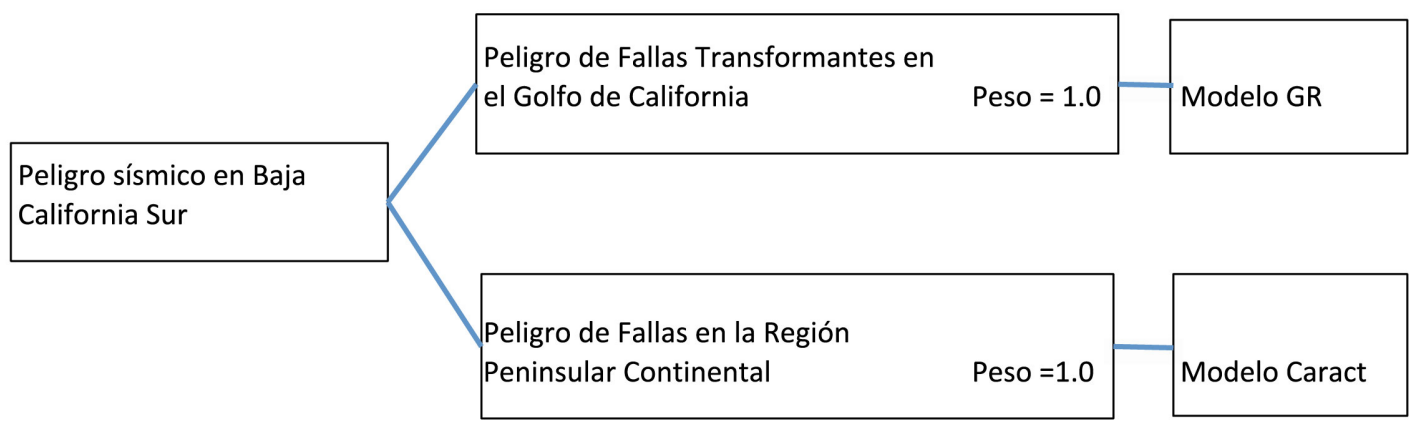

Figura 2. Ponderación probabilística para el cálculo total de peligro sísmico. 
Sólo las estructuras con riesgo potencial (fallas con longitud mayor a $10 \mathrm{~km}$ ) fueron utilizadas para el análisis de peligro sísmico posterior. Sin embargo, toda la base de datos de las estructuras aportó la información regional que se requiere para integrar los rasgos estructurales en el estudio de peligro sísmico. En la Figura 4 se muestra el sistema de fallas que se identificaron en este estudio.

Después de integrar los pasos descritos anteriormente, se procedió a preparar el marco formal basado en los códigos HAZMAP del USGS. Además se utilizó un código de

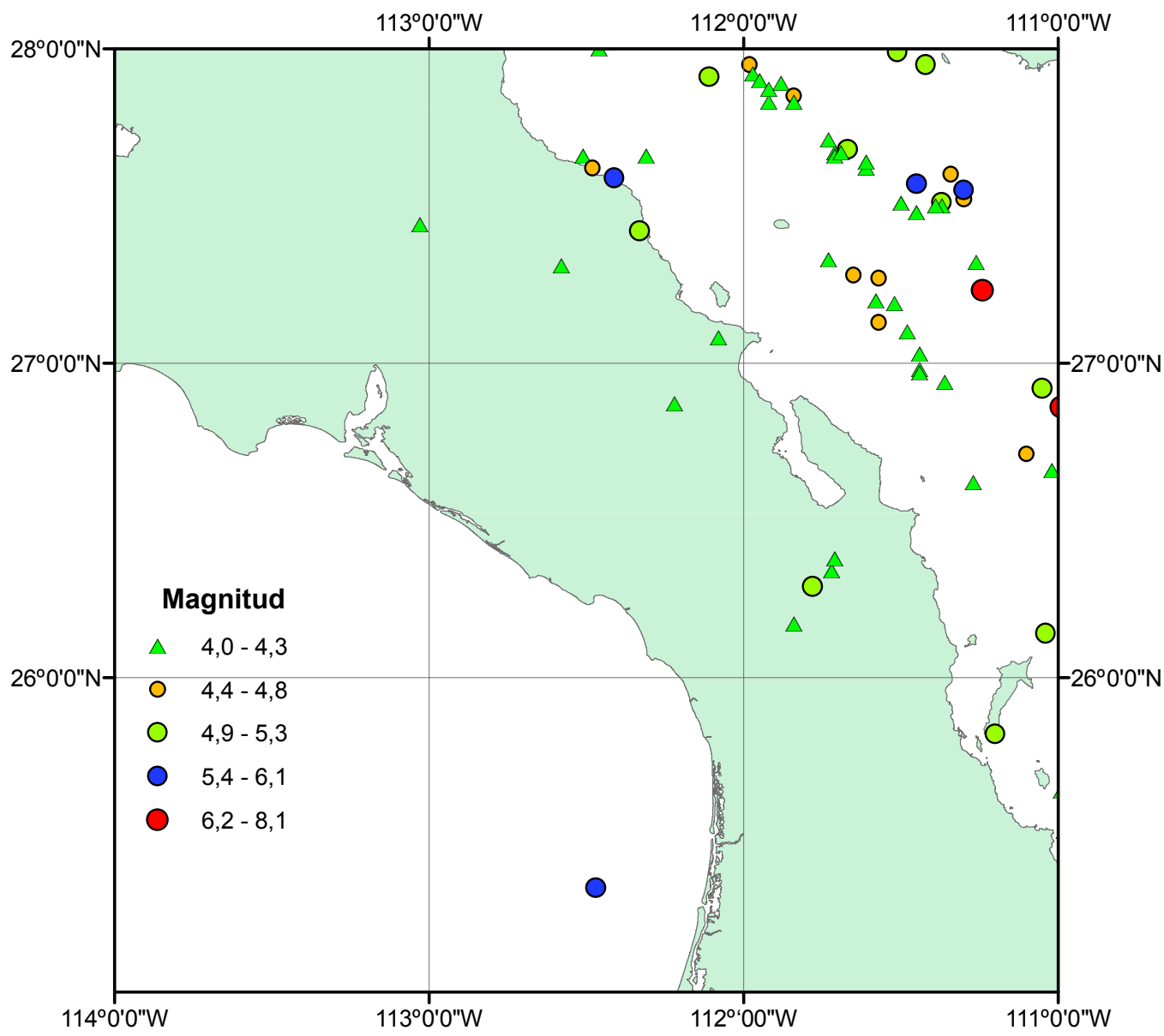

Figura 3. Localización de los eventos del catálogo del SSN. Los eventos dentro de la península son de baja magnitud $(<4.3 \mathrm{M})$. El catálogo sísmico fue usado para la estimación de los parámetros $b$ de la región sismotectónica del Golfo de California. Los eventos de la península de California indican la presencia de las fallas cuaternarias las cuales se analizan mediante modelos de falla característica.

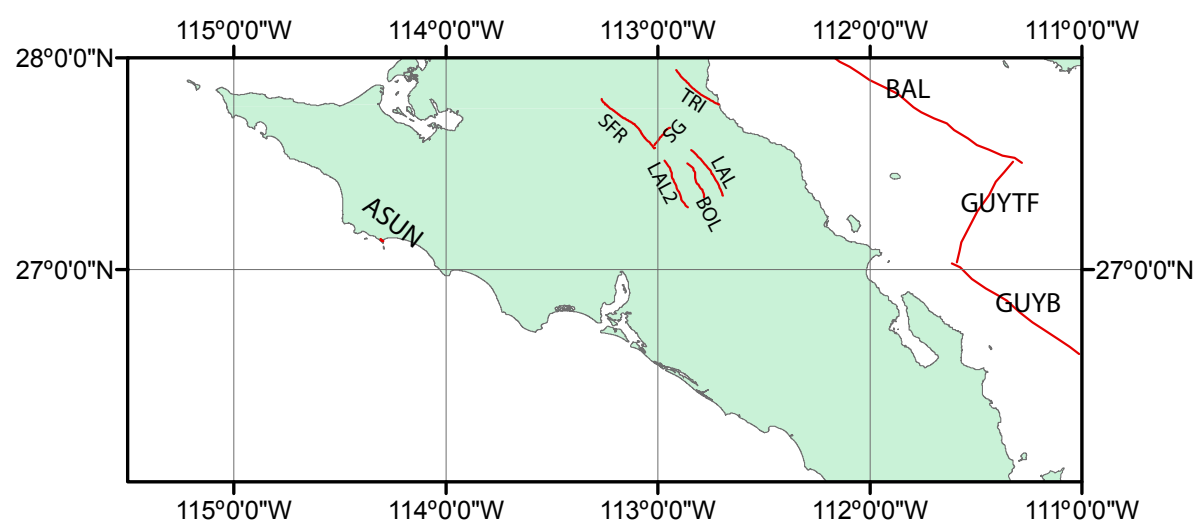

Figura 4. Localización de las principales fallas estudiadas. Fallas transformes Ballenas, BAL; Guaymas, GUYB; Cuencas Guaymas, GUYTF. Fallas Peninsulares Trinidad, TRI; San Francisco, SFR; San Gregorio, SG; Lomas Altas 1, LAL; Lomas Altas 2, LAL2; Boleo, BOL; Asunción, ASUN. 
Teoría de las Vibraciones Aleatorias (Boore, 1983) para la preparación de las curvas de atenuación para la provincia extensional del Golfo de California.

\section{Atenuación regional y escalamiento}

Se utilizaron algoritmos que utilizan procesos estocásticos basados en la teoría de las vibraciones aleatorias para construir las tablas de atenuación. Estos algoritmos utilizan modelos de fuente tipo Brune (1970), con funciones segmentadas de valores de expansión geométrica y de atenuación $Q$ particulares. Los parámetros que se utilizaron para la construcción de este mapa de peligro sísmico se muestran en la Tabla 2 (Modelo BCS). El análisis detallado en el cálculo de las funciones predictivas se describe en Ortega y González (2007).

Se prepararon las curvas de atenuación con base en tablas de magnitud-distancia. Las curvas generadas se muestran en la Figura 5.

Los resultados son usados en los programas de peligro sísmico; cabe destacar que los valores de atenuación para BCS fueron comparados con los valores de NGA (NGA, 2014) para un sitio con condiciones de suelo de $\mathrm{V}_{\mathrm{s} 30}=$ $760 \mathrm{~m} / \mathrm{s}$ y se observa que ambas curvas de atenuación son equivalentes debido a que tienen parámetros $\mathrm{Q}, \eta \mathrm{y}$ de expansión geométrica similares. Por esta razón se procedió a elaborar un árbol lógico que cubra las leyes de atenuación como se muestra en el esquema de la Figura 6.

El programa de cálculo de peligro sísmico fue preparado usando un esquema probabilístico en la magnitud máxima con una incertidumbre de $M_{\max } \pm 0.2$ con pesos de 0.8 para la $M_{\max }$ calculada por Wells y Coppersmith (1994) y 0.1 para los intervalos superiores e inferiores como se muestra en la Figura 7. Este esquema permite incluir incertidumbres en la longitud de la falla, debido a que en esta zona la cartografía de las fallas activas es variable porque en varios casos la longitud está inferida.

\section{Atenuación regional y escalamiento}

El cálculo final se resume con las siguientes consideraciones:

- La condición de sitio de suelo específico para diseño de ingeniería es de $\mathrm{v} 30=760 \mathrm{~m} / \mathrm{s}$.

- Los modelos de atenuación son NGA $40 \%$ y BCS $60 \%$.

- La información de sismicidad fue utilizada con el modelo Gutenberg-Richter solo para las fallas activas del Golfo de California usando los parámetros $a$ y $b$. Para la región peninsular continental solo se usó la información de tasa de desplazamientos.

- Los modelos probabilísticos para magnitud máxima utilizaron una incertidumbre de $\pm 0.2 \mathrm{M}$.

- Los valores de atenuación para BCS fueron basados en Ortega y González (2007).

- Se realizaron cálculos de periodos de retorno que corresponden a $10 \%, 5 \%$ y $2 \%$ de excedencia a 50 , 100 y 200 años.

A fin de ejemplificar la importancia de cada falla,

Tabla 2. Parámetros de escalamiento para las funciones predictivas. $\kappa_{\text {eff }}$ es el factor que determina la atenuación de sitio a altas frecuencias, $\beta$ es la velocidad de corte promedio en la corteza, $\rho$ es la densidad, $\mathrm{g}(\mathrm{r})$ es la función de expansión geométrica de la propagación de las ondas sísmicas, es la expresión de la velocidad de corte promedio en roca dura para efecto de amplificación, donde GEN97 es el modelo genérico de amplificación de sitio descrito por Boore y Joyner (1997).

\begin{tabular}{|c|c|c|c|c|c|c|c|c|c|}
\hline & \multicolumn{4}{|c|}{ HIGHAT } & \multicolumn{5}{|c|}{ LOWAT } \\
\hline$\kappa_{\mathrm{eff}}$ & \multicolumn{4}{|c|}{0.06} & \multicolumn{5}{|c|}{0.016} \\
\hline$Q(f)$ & \multicolumn{4}{|c|}{$180 f^{0.32}$} & \multicolumn{5}{|c|}{$380 f^{0.10}$} \\
\hline $\begin{array}{c}\beta \\
{[\mathrm{km} / \mathrm{s}]}\end{array}$ & \multicolumn{4}{|c|}{3.5} & \multicolumn{5}{|c|}{3.5} \\
\hline $\begin{array}{c}\rho \\
{\left[\mathrm{gr} / \mathrm{cm}^{3}\right]}\end{array}$ & \multicolumn{4}{|c|}{2.8} & \multicolumn{5}{|c|}{2.8} \\
\hline$g(r)$ & $\left\{\begin{array}{lc}r^{-1.0} & 0 \\
r^{-0.2} & 40 \\
r^{-0.5} & 100\end{array}\right.$ & $\begin{array}{l}<\quad r \\
<\quad r \\
<\quad r\end{array}$ & $\begin{array}{l}\leq \\
\leq \\
\leq\end{array}$ & $\begin{array}{c}40 \\
100 \\
180\end{array}$ & $\left\{\begin{array}{l}r^{-1.1} \\
r^{-0.3} \\
r^{-0.8} \\
r^{-0.5}\end{array}\right.$ & $\begin{array}{c}0 \\
40 \\
70 \\
100\end{array}$ & $\begin{array}{l}<r \\
<\quad r \\
<r \\
<r\end{array}$ & $\begin{array}{l}\leq \\
\leq \\
\leq \\
\leq\end{array}$ & $\begin{array}{c}40 \\
70 \\
100 \\
180\end{array}$ \\
\hline $\bar{V}(f)$ & \multicolumn{4}{|c|}{ GEN97 } & \multicolumn{5}{|c|}{ GEN97 } \\
\hline $\begin{array}{c}\Delta \sigma \\
\text { [bars] }\end{array}$ & \multicolumn{4}{|c|}{40} & \multicolumn{5}{|c|}{40} \\
\hline
\end{tabular}




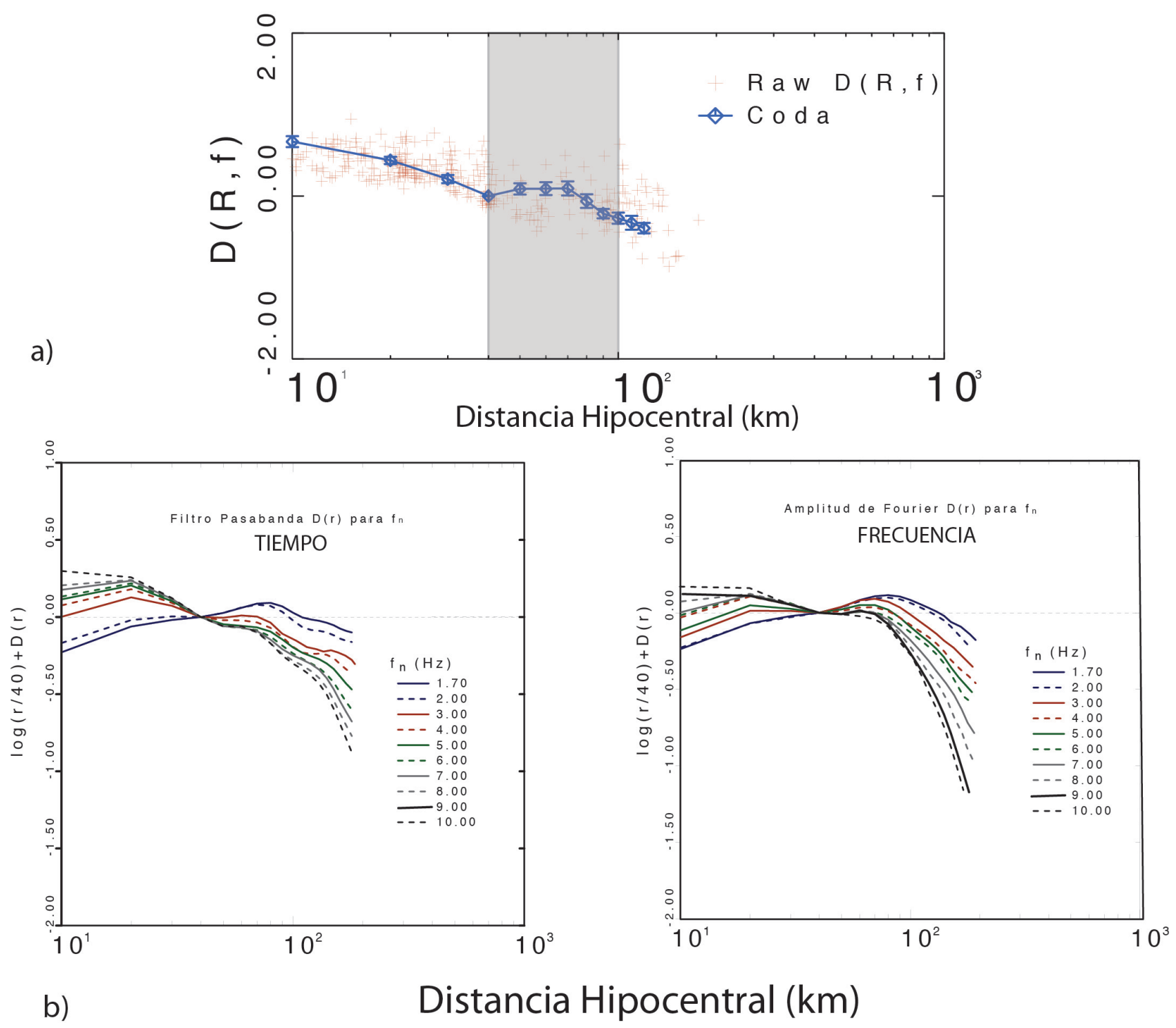

Figura 5. a) Resultados de regresión de la curva de atenuación. El intervalo supercrítico entre los 40 y $100 \mathrm{~km}$ se encuentra sombreado. b) En la parte inferior se muestra la modelación estocástica mediante teoría de las variaciones aleatorias de diferentes frecuencias.

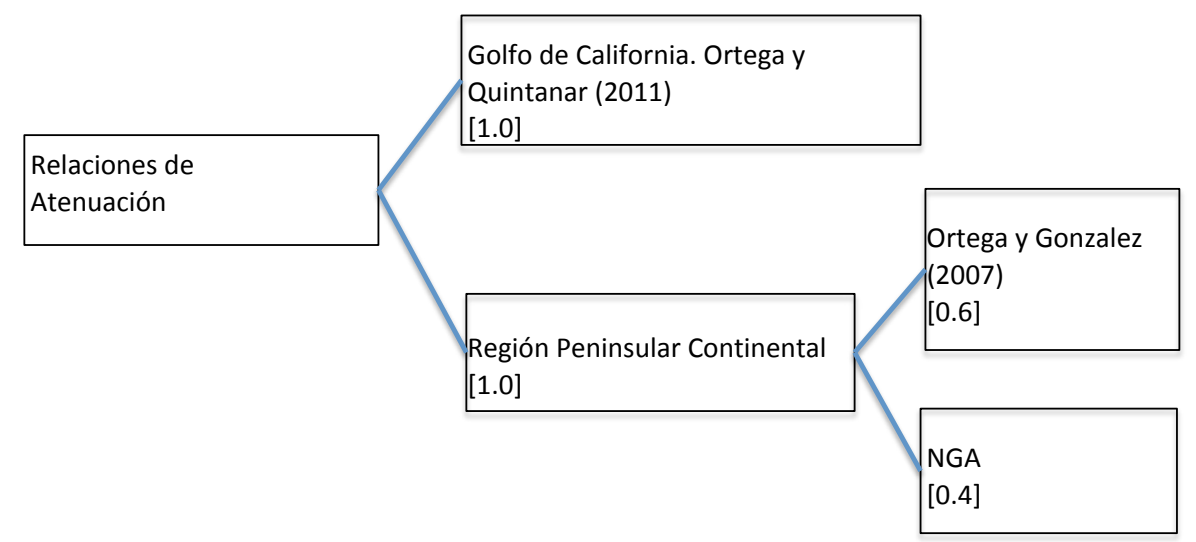

Figura 6. Árbol lógico para las curvas de atenuación estimadas en el peligro sísmico. Los valores en la parte inferior corresponden al peso probabilístico estimado. 


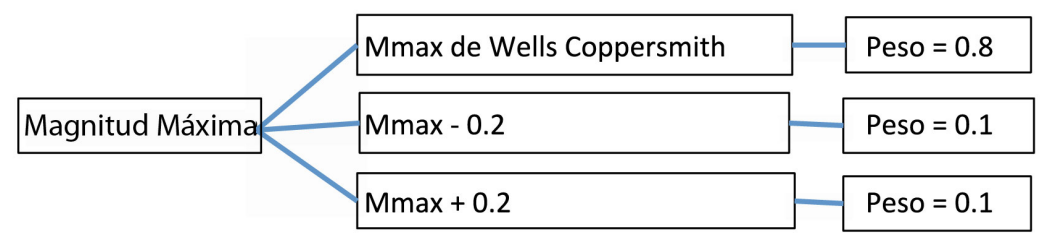

Figura 7. Árbol lógico para estimar la magnitud máxima.

incluyendo fallas pequeñas, se elaboraron dos modelos. El modelo A utiliza todas las fallas (1-7 Tabla 1), mientras que el modelo B descarta la falla Asunción, la cuál es la más corta y se encuentra aislada en la parte Este. Antes de 2007 se desconocía la existencia de esta falla y fue añadida basándose en la actividad reciente. Estos dos modelos los hemos comparado en las figuras de 8 a 13, representando el peligro sísmico para diferentes periodos de retorno. Se observó que para el sitio de Bahía Asunción, el peligro sísmico es diferente sólo cuando el periodo de retorno es mayor a 2527 años. Este resultado no es de sorprender porque se supone una distribución de Poisson para el cálculo de la probabilidad. En secciones siguientes se describirá el efecto que tiene usar periodos de retorno conservadores e incluso no incluir fallas activas para un ejemplo en el diseño de espectros de respuesta de sitio.

Las curvas de atenuación muestran una tendencia que refleja el patrón de recurrencia que rige el modelo característico basado en la razón de desplazamiento de las fallas regionales; es decir, el periodo de retorno se define como per_retorno $=M_{O} / M_{O C}$. Como ejemplo, para la Falla San Francisco y la Falla Trinidad estos periodos de retorno son aproximadamente de 1700 y 1900 años respectivamente. Si se comparan estos periodos de retorno con los estudios de peligro sísmico que consideran periodos de 474, 975 y 2475 años, entonces, las curvas de peligro sísmico en estos intervalos sólo consideran un ciclo en su intervalo de recurrencia. Es decir, solo se manifiestan para el caso de 2475 años ( $2 \%$ de probabilidad en 50 años) de la figura 8. Evidentemente, a periodos mayores, las curvas de peligro sísmico se manifiestan con mayor variación en los valores esperados de PGA. Este resultado es consecuencia de los valores utilizados de la tasa de deslizamiento anual, la cual es del orden de un milímetro al año (Tabla 1).

Se observa que las relaciones de atenuación son similares a la nueva generación de curvas de atenuación (NGA) para la estimación de PGA a V30 $=760 \mathrm{~m} / \mathrm{s}$. Por esta razón se utilizó la ponderación de 0.6 para las curvas de BCS y 0.4 para NGA. Este tipo de sitios es adecuado para el diseño de ingeniería estructural. La tabla 3 resume los valores de PGA para el sitio de Bahía Asunción.

\section{Aplicación a Espectros de Respuesta de Sitio}

Se calculó el espectro de respuesta de sitio basado en el Manual de Diseño por Sismo de la Comisión Federal de Electricidad (CFE, 2008). Se utilizaron los datos de velocidad de corte de un sitio ubicado en las coordenadas $27.143^{\circ} \mathrm{N}-114.294^{\circ} \mathrm{W}$, los cuales fueron obtenidos mediante dispersión de ondas superficiales. Este caso ejemplifica claramente la forma en la cual se aplica el estudio de peligro sísmico para diseño de obras civiles. Además, en este sitio se han registrado aceleraciones extremas de 600 gales durante una intensa actividad ocurrida en 2007, (Munguía, 2014, comunicación escrita). Por esta razón se procedió a comparar los resultados del análisis de peligro sísmico, con las observaciones de campo. En la Figura 14 se observa una gráfica del perfil de velocidades de corte utilizando la técnica de dispersión de ondas superficiales. Se utilizaron geófonos de $4.5 \mathrm{~Hz}$ en un arreglo MASW con espaciamiento de 7 metros. Los resultados fueron comparados con valores de penetración estándar. La descripción completa de esta metodología está fuera del alcance de este artículo y se presenta en CFE (1998). Es importante destacar que a partir del modelo de velocidades de corte y con los valores de PGA de la tabla 3 se puede construir el espectro de respuesta de sitio, el cual está representado gráficamente por un trapecio donde agrupa los periodos de diseño. Se construyeron los espectros de respuesta para este sitio utilizando los datos de la tabla 3 y el perfil de velocidades (Figura 15). En la figura se presentan 5 espectros de respuesta, destacando que solo los periodos de retorno $2 \%$ en $100,5 \%$ en 200 y $2 \%$ en 200 son diferentes al resto de los valores de la tabla 3. Para todos los demás valores, el espectro es el mismo al seguir las recomendaciones de diseño por sismo; sin

Tabla 3. Valores de PGA para los modelos MOD A y MOD B.

\begin{tabular}{lccc}
\hline Excedencia (\%) & $\begin{array}{c}\text { Periodo retorno } \\
\text { (años) }\end{array}$ & $\begin{array}{c}\text { MOD A } \\
\text { (pga) } \\
{[\mathrm{g}]}\end{array}$ & $\begin{array}{c}\text { MOD B } \\
(\mathrm{pga}) \\
{[\mathrm{g}]}\end{array}$ \\
\hline $10 \%$ en 50 & 475 & 0.013 & 0.016 \\
$5 \%$ en 50 & 976 & 0.022 & 0.027 \\
$2 \%$ en 50 & 2475 & 0.033 & 0.075 \\
$10 \%$ en 100 & 950 & 0.022 & 0.026 \\
$5 \%$ en 100 & 1950 & 0.03 & 0.048 \\
$2 \%$ en 100 & 4950 & 0.04 & $\mathbf{0 . 3 4 4}$ \\
$10 \%$ en 200 & 1898 & 0.029 & 0.046 \\
$5 \%$ en 200 & 3900 & 0.037 & $\mathbf{0 . 2 7 7}$ \\
$2 \%$ en 200 & 9900 & 0.049 & $\mathbf{0 . 5 1 7}$ \\
\hline
\end{tabular}



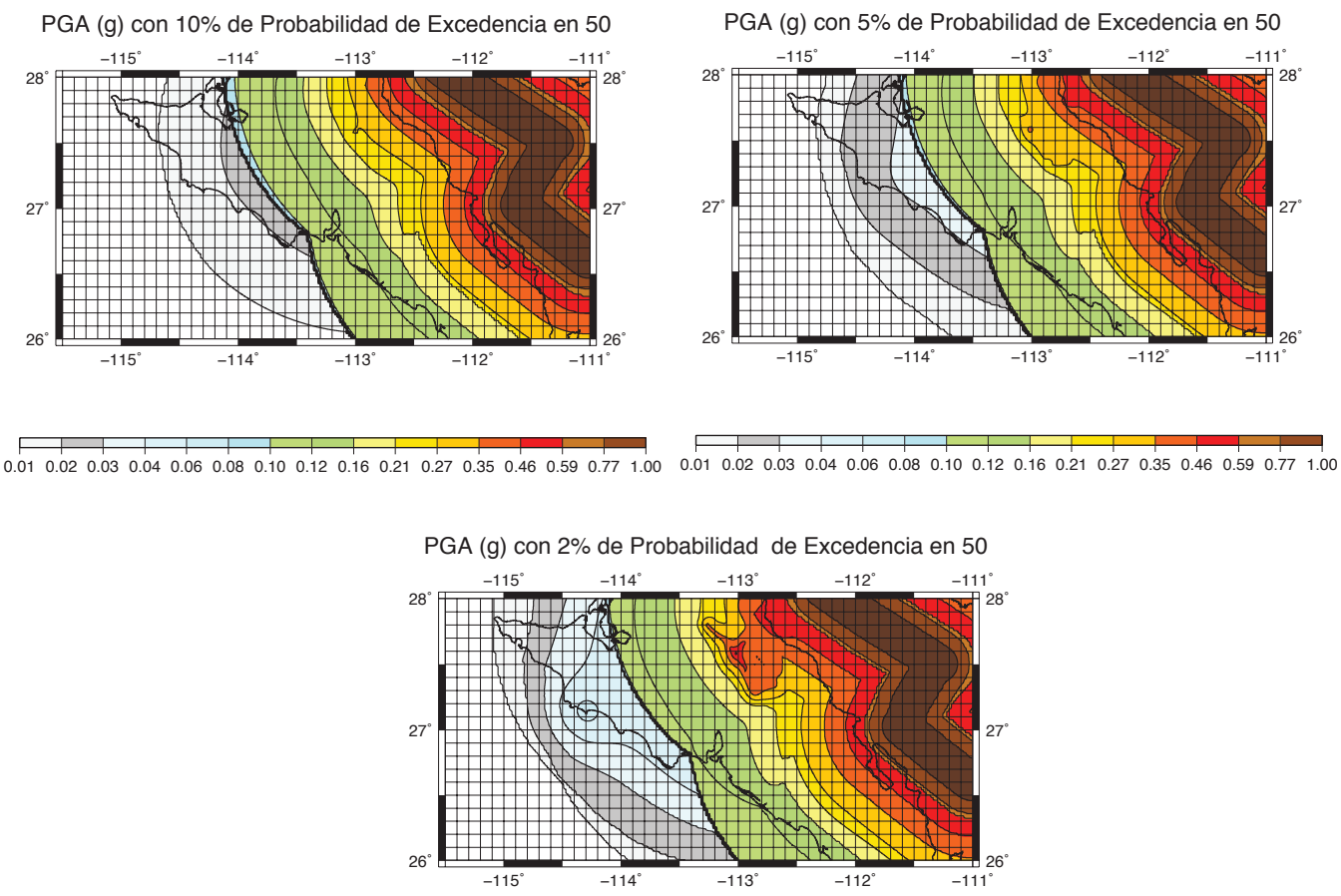

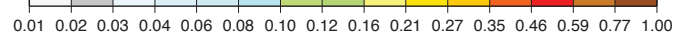

Figura 8. Peligro sísmico total del modelo A (todas las fallas) para el cálculo de espectros de sitio calculados para Vs $30=760 \mathrm{~m} / \mathrm{s}$ utilizando la distribución de Poisson de probabilidad. a) Peligro para probabilidad de $10 \%$ de excedencia en 50 años, b) Peligro para probabilidad de $5 \%$ en 50 años y c) Peligro para $2 \%$ en 50 años. Los periodos de retorno equivalen a 475,975 y 2475 años
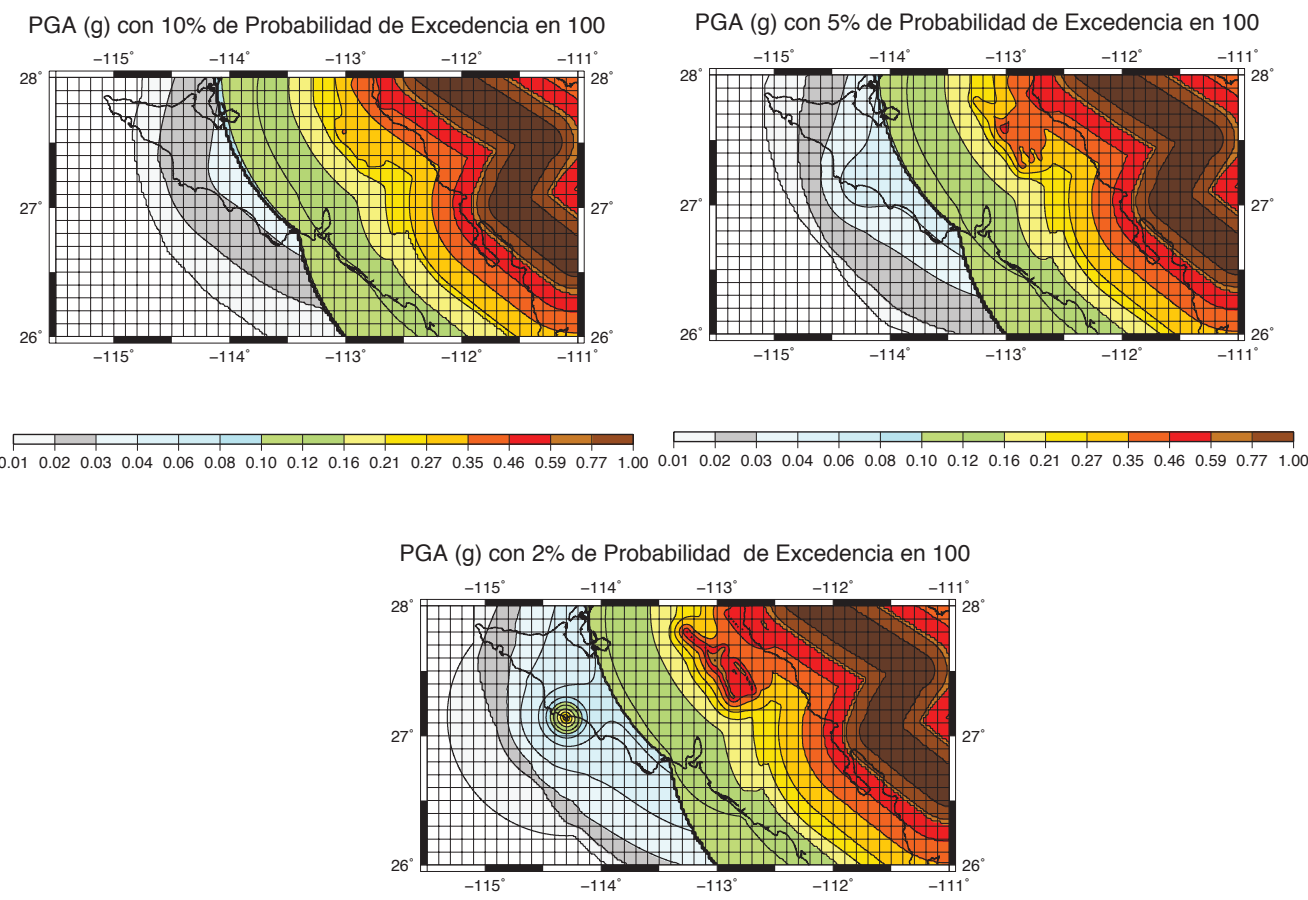

$\begin{array}{llllllllllllllll}0.01 & 0.02 & 0.03 & 0.04 & 0.06 & 0.08 & 0.10 & 0.12 & 0.16 & 0.21 & 0.27 & 0.35 & 0.46 & 0.59 & 0.77 & 1.00\end{array}$

Figura 9. Peligro sísmico total del modelo A (todas las fallas) para el cálculo de espectros de sitio calculados para Vs30=760 m/s utilizando la distribución de Poisson de probabilidad. a) Peligro para probabilidad de $10 \%$ de excedencia en 100 años, b) Peligro para probabilidad de 5\% en 100 años y c) Peligro para $\%$ en 100 años. Los periodos de retorno equivalen a 950, 1950 y 4950 años. 


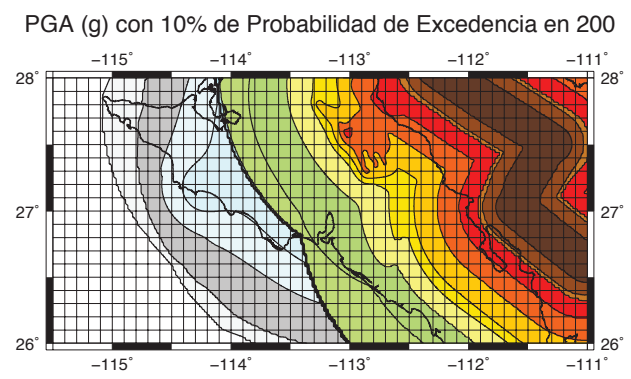

PGA (g) con 5\% de Probabilidad de Excedencia en 200

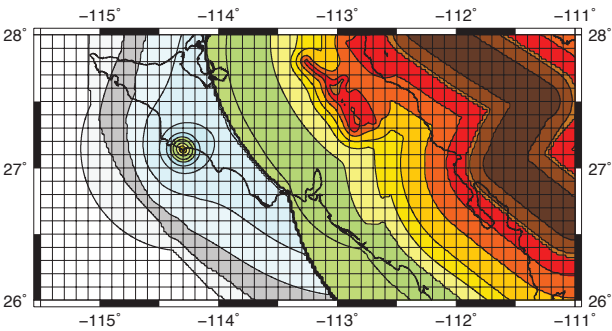

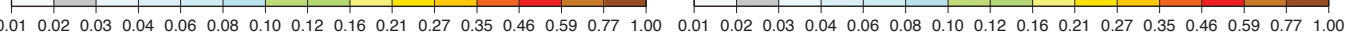

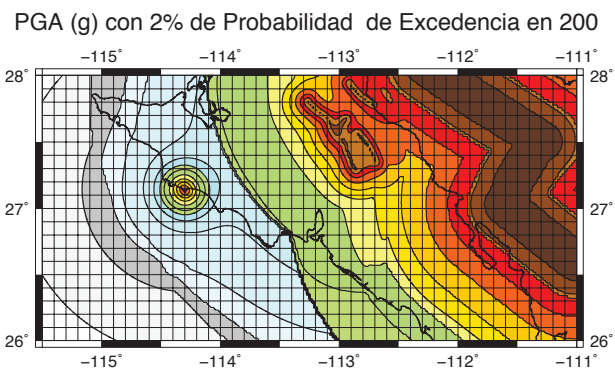

$\begin{array}{lllllllllllllllll}0.01 & 0.02 & 0.03 & 0.04 & 0.06 & 0.08 & 0.10 & 0.12 & 0.16 & 0.21 & 0.27 & 0.35 & 0.46 & 0.59 & 0.77 & 1.00\end{array}$

Figura 10. Peligro sísmico total del modelo A (todas las fallas) para el cálculo de espectros de sitio calculados para Vs $30=760 \mathrm{~m} / \mathrm{s}$ utilizando la distribución de Poisson de probabilidad. a) Peligro para probabilidad de $10 \%$ de excedencia en 200 años, b) Peligro para probabilidad de $5 \%$ en 200 años y c) Peligro para $2 \%$ en 200 años. Los periodos de retorno equivalen a 1900, 3900 y 9900 años.

PGA (g) con 10\% de Probabilidad de Excedencia en 50

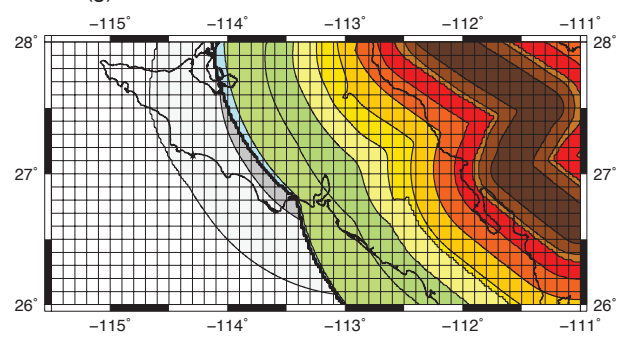

$\begin{array}{llllllllllllllllll}0.01 & 0.02 & 0.03 & 0.04 & 0.06 & 0.08 & 0.10 & 0.12 & 0.16 & 0.21 & 0.27 & 0.35 & 0.46 & 0.59 & 0.77 & 1.00 & 0\end{array}$
PGA (g) con 5\% de Probabilidad de Excedencia en 50

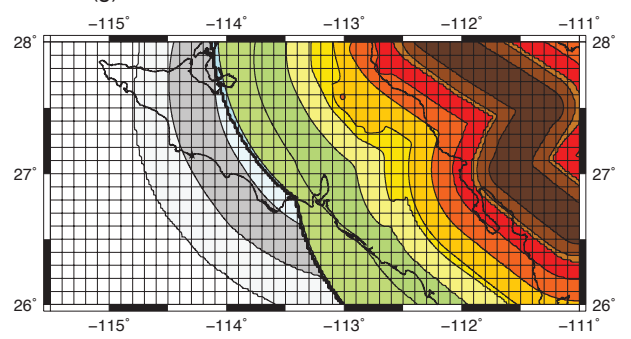

$\begin{array}{lllllllllllllllllllllll}01 & 0.02 & 0.03 & 0.04 & 0.06 & 0.08 & 0.10 & 0.12 & 0.16 & 0.21 & 0.27 & 0.35 & 0.46 & 0.59 & 0.77 & 1.00\end{array}$

PGA (g) con $2 \%$ de Probabilidad de Excedencia en 50

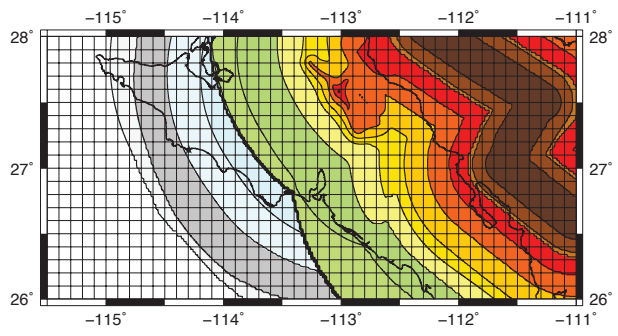

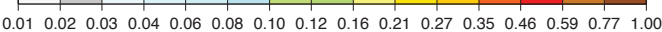

Figura 11. Peligro sísmico total del modelo B (eliminando Falla Asunción) para el cálculo de espectros de sitio calculados para Vs30=760 m/s utilizando la distribución de Poisson de probabilidad. a) Peligro para probabilidad de $10 \%$ de excedencia en 50 años, b) Peligro para probabilidad de $5 \%$ en 50 años y c) Peligro para $2 \%$ en 50 años. Los periodos de retorno equivalen a 950, 1950 y 4950 años. 
PGA (g) con $10 \%$ de Probabilidad de Excedencia en 100

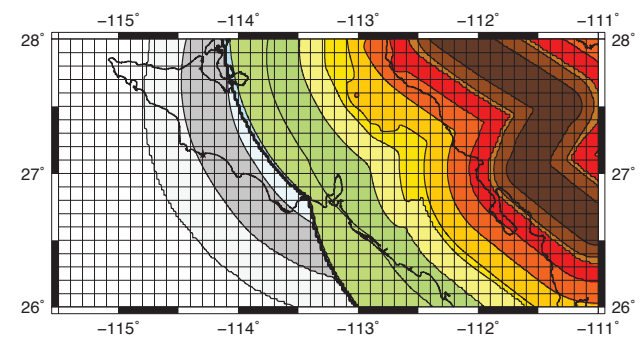

PGA (g) con 5\% de Probabilidad de Excedencia en 100

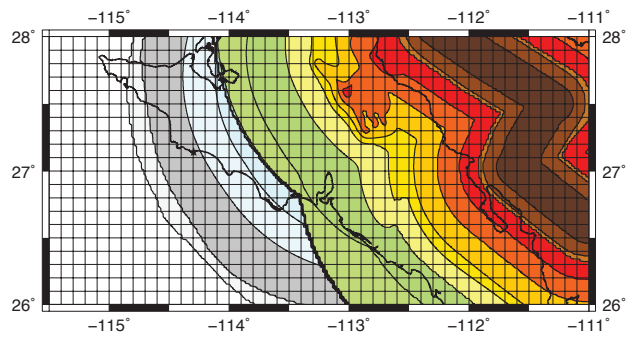

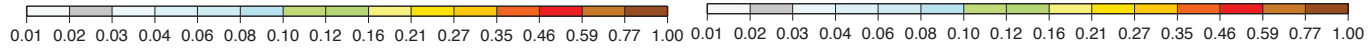

PGA (g) con 2\% de Probabilidad de Excedencia en 100

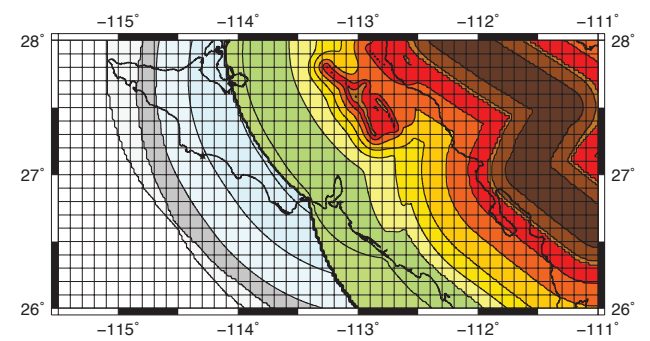

$\begin{array}{lllllllllllllllll}0.01 & 0.02 & 0.03 & 0.04 & 0.06 & 0.08 & 0.10 & 0.12 & 0.16 & 0.21 & 0.27 & 0.35 & 0.46 & 0.59 & 0.77 & 1.00\end{array}$

Figura 12. Peligro sísmico total del modelo A (eliminando Falla Asunción) para el cálculo de espectros de sitio calculados para Vs $30=760 \mathrm{~m} / \mathrm{s}$ utilizando la distribución de Poisson de probabilidad. a) Peligro para probabilidad de $10 \%$ de excedencia en 100 años, b) Peligro para probabilidad de $5 \%$ en 100 años y c) Peligro para 2\% en 100 años. Los periodos de retorno equivalen a 1900, 3900 y 9900 años.

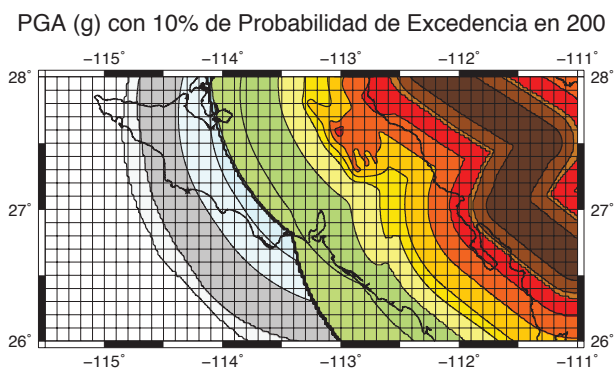

PGA (g) con 5\% de Probabilidad de Excedencia en 200

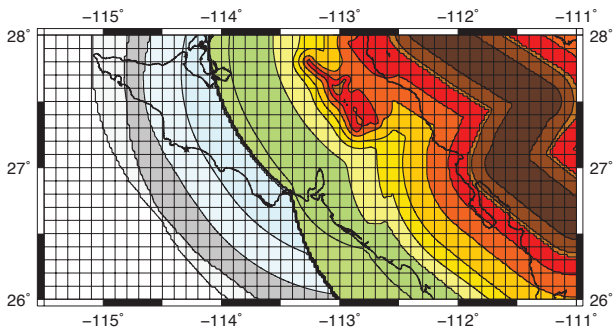

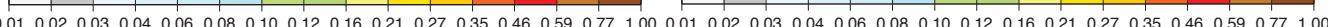

PGA (g) con $2 \%$ de Probabilidad de Excedencia en 200

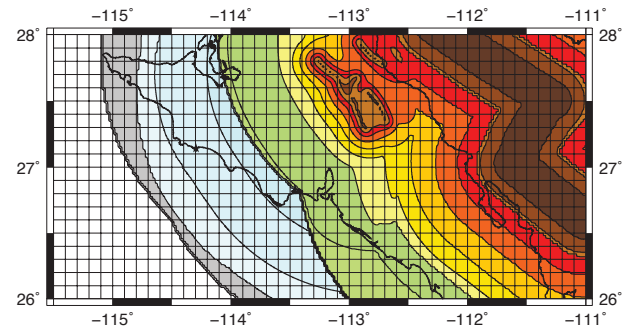

$\begin{array}{lllllllllllllllllll}0.01 & 0.02 & 0.03 & 0.04 & 0.06 & 0.08 & 0.10 & 0.12 & 0.16 & 0.21 & 0.27 & 0.35 & 0.46 & 0.59 & 0.77 & 1.00\end{array}$

Figura 13. Peligro sísmico total del modelo B (eliminando Falla Asunción) para el cálculo de espectros de sitio calculados para Vs $30=760 \mathrm{~m} / \mathrm{s}$ utilizando la distribución de Poisson de probabilidad. a) Peligro para probabilidad de $10 \%$ de excedencia en 200 años, b) Peligro para probabilidad de 5\% en 200 años y c) Peligro para 2\% en 200 años. Los periodos de retorno equivalen a 1900, 3900 y 9900 años. 
embargo, es común realizar un factor de seguridad para las llamadas estructuras tipo A, estas estructuras representan mayor vulnerabilidad debido a la necesidad de ser resistentes en caso de sismos. En la figura 15 se muestran todos los espectros de respuesta de este sitio y el valor de amplitud del máximo espectral del sismo del 1 de Noviembre del

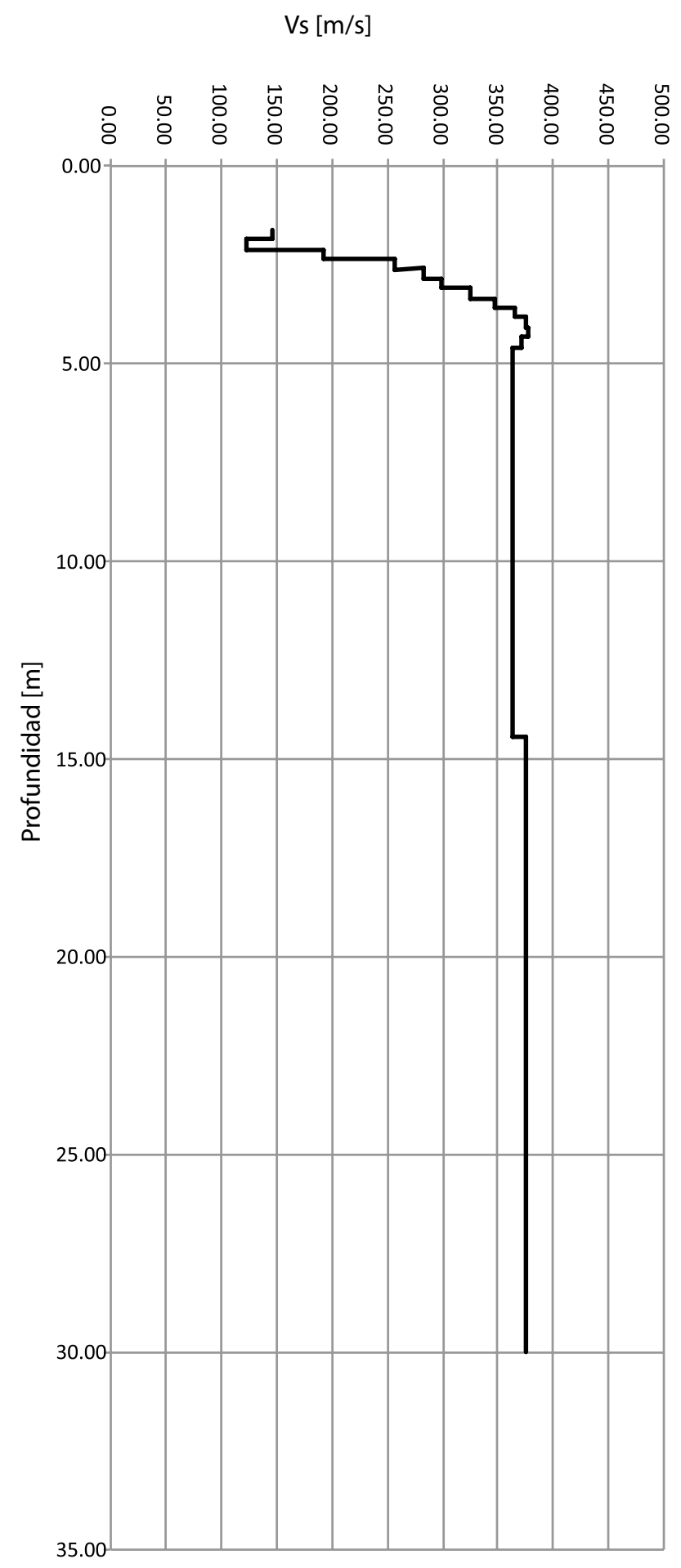

Figura 14. Modelo de velocidades obtenido para el sitio de Bahía Asunción.
2006. Es interesante notar que cuando no está considerada la falla Asunción o cuando los valores de recurrencia son menores a 2500 años, el espectro de respuesta de sitio siempre es el menor, lo cual se representa en la figura 15 . Solamente tres casos son aceptables y estos pertenecen a los modelos de recurrencia que implican la actividad de la falla característica Asunción. Por esta razón es importante incluir la información de las fallas del modelo característico para el diseño de estructuras; es decir, es importante escoger el periodo de retorno con base en la información geológica de la tabla 1 y no utilizar periodos de retorno establecidos por diversas normas internacionales las cuales no siempre están bien sustentadas.

\section{Discusión y Conclusiones}

Se observa que el peligro sísmico está controlado por las fallas transformantes para periodos de recurrencia cortos, mientras que las fallas peninsulares controlan el peligro de periodos largos. Se demostró que la información de las fallas activas cercanas a los elementos para redefinir los períodos de retorno de los sitios de mayor amenaza. Es importante tener estudios completos de todas las fuentes sísmicas para la preparación de mapas de peligro sísmico. Además, existen algunas consideraciones que pueden ser mal interpretadas, como por ejemplo el modelo característico. Este modelo, a diferencia del modelo de sismicidad de Gutenberg-Richter, supone que las fallas rompen con una magnitud relativamente constante a intervalos también constantes. En general el modelo característico está bien fundamentado en estudios de paleosismología y ha sido útil en la preparación de mapas de peligro sísmico (Frankel, 1995). Sin embargo, existen problemas en la interpretación y en los esquemas de elaboración de mapas de peligro sísmico. Por ejemplo, el periodo de recurrencia en los códigos de peligro sísmico, supone que la falla asignada a este modelo tendrá su siguiente rompimiento solamente hasta que se cumpla el periodo de retorno, esto significa que si se designa una fuente cuya recurrencia es de 0.001 (mil años de periodo de retorno), entonces en los mapas de peligro sísmico no se observa la peligrosidad de la falla para periodos de retorno menores a éstos 1000 años. Esta observación es correcta solo si los periodos son de varios ordenes de magnitud más grande, es decir, si los mapas fueran de periodos de decenas de miles de años. Pero para los casos típicos de mapas de peligro sísmico se requiere de mayores datos, por ejemplo, si se conoce que la falla estudiada ha permanecido inactiva por 900 años, entonces es importante incluir dicha información en los mapas. Esta dependencia temporal no está incluida aún en los mapas tradicionales de peligro sísmico. Muchos esfuerzos que se han hecho recientemente están enfocados en añadir información temporal a los mapas de peligro sísmico (Stein et al., 2006). Por otro lado, esta información de dependencia temporal es inconsistente con el modelo de distribución de 


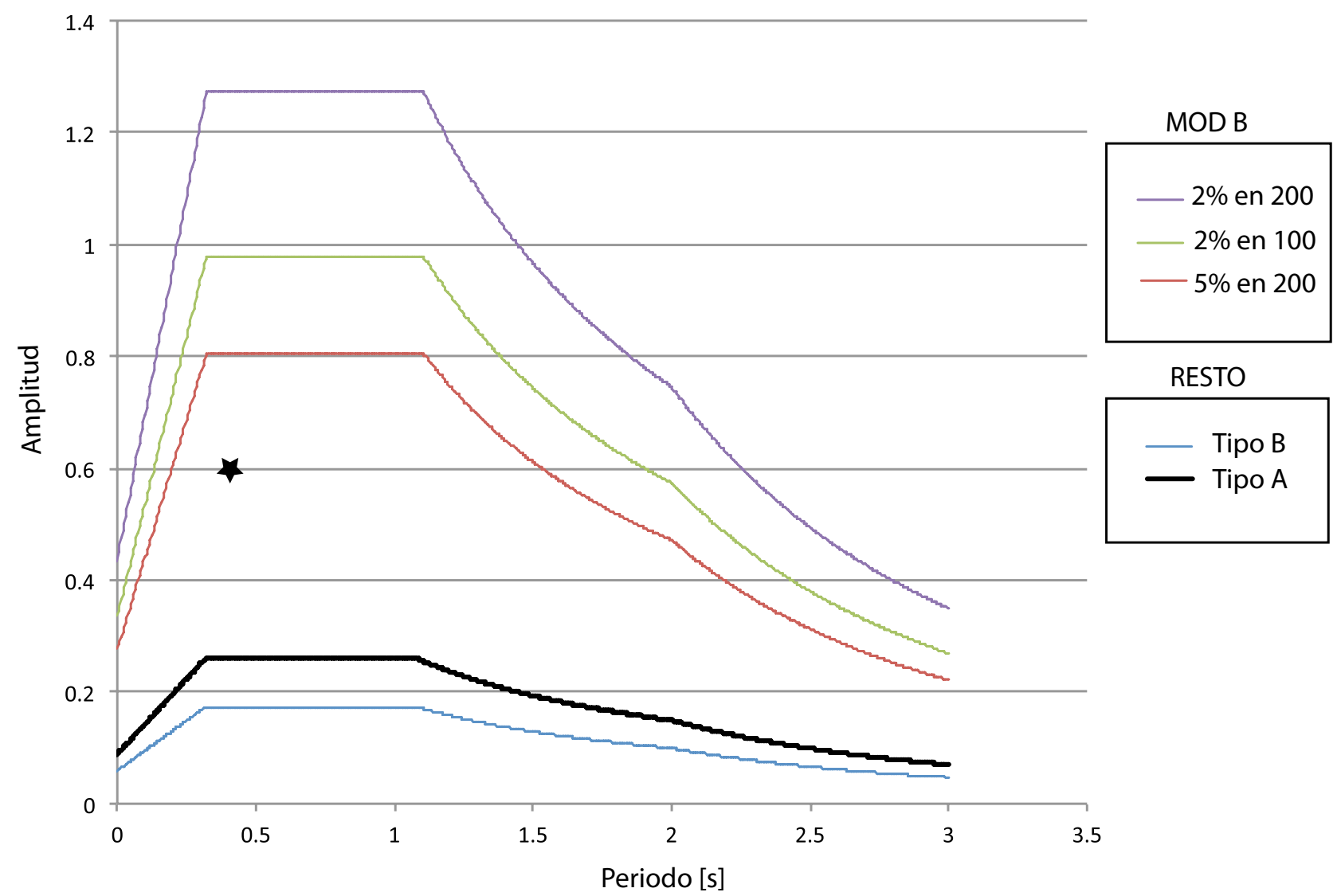

Figura 15. Espectros de respuesta de sitio calculados usando el Manual de Diseño por Sismos de la Comisión Federal de Electricidad con datos del perfil de velocidad de la Figura 13 y los valores PGA de la tabla 3. En la parte superior se destacan los tres valores resaltados de la tabla 3, en la parte inferior se muestran el resto de los valores en los cuales coincide el mismo espectro. Además se muestra para este último caso, el espectro de respuesta para estructuras tipo B, y el espectro para estructuras tipo A, este último se calcula multiplicando por 1.5. El valor de máximo espectral obtenido en 2006 en ese sitio está representado por una estrella.

Poisson, el cual está basado en independencia temporal por lo que los mapas tradicionales de peligro sísmico suponen que en cualquier instante, el mapa es válido. Este artículo nos permite destacar la inconsistencia de los dos modelos y proponer por lo menos una solución sencilla para sitios en los cuales el peligro sísmico depende de fallas características, suponiendo que existe evidencia geológica de que dichas fallas están activas. Entonces para estructuras esenciales (Grupo A) el periodo de retorno mínimo debe ser por lo menos igual a la recurrencia de la falla característica. Es decir, en este ejemplo, a pesar que la estructura tipo A aconseje un periodo de retorno de 2475 ( $2 \%$ en 50), es claro que se necesita por lo menos un periodo de 2700 años para poder diseñar espectros seguros. Esto tiene una repercusión importante en los códigos de construcción porque a pesar de usar factores de seguridad para estructuras tipo A, no es suficiente si no se ha incluido un periodo de retorno que represente la amenaza potencial de la falla. Es claro que se necesitan modelos consistentes entre un análisis GutenbergRichter y un análisis de sismos característicos. Estos últimos deben de ser la base para decidir el periodo de retorno de las estructuras esenciales. Para el caso del centro de Baja
California y en especial en la zona de Bahía Asunción, es necesario utilizar los periodos de retorno mayores a 2700 años y no los estándares internacionales. En cada sitio se debe de encontrar el periodo de retorno dependiendo de las fallas locales y sus parámetros de desplazamiento. De esta forma el espectro de diseño de sitio es adecuado para asegurar estructuras sismo-resistentes.

El reto más grande para la elaboración de mapas de peligro sísmico consiste en localizar las fallas activas, sobre todo en valles sedimentarios como el Desierto del Vizcaíno donde las estructuras se encuentran cubiertas. Existen técnicas directas e indirectas, además hay soluciones alternas que pueden inferir la existencia de fallas activas ocultas, por ejemplo, usando redes geodésicas de GPS para calcular los desplazamientos relativos y calcular la razón de momento total estimada en la región. Este tipo de inferencias han sido exitosamente probadas en lugares como Nueva Zelanda donde se ha considerado el déficit de razón de momento en los mapas de peligro sísmico, y se han mejorado sustancialmente dichos mapas. Resulta evidente que a pesar de que los modelos de Gutenberg-Richter y Característico presentan problemas en su interpretación, 
sobre todo al integrarlos en un mismo mapa, el resultado final puede asegurar un diseño adecuado cuando se han considerado cuidadosamente todas las fuentes sísmicas.

\section{Agradecimientos}

Los autores agradecen la información de mecánica de suelos de Geobaja Ingeniería. Queremos agradecer la ayuda de Víctor Manuel Espíndola por proporcionar datos de sismicidad y a Luis Munguía Orozco por sus comentarios. Agradecemos a Hugo Monzalve por su revisión. Agradecemos especialmente a un revisor anónimo por la detallada y cuidadosa revisión de la redacción del manuscrito.

\section{References}

Anderson, J.G., 1979, Estimating the seismicity from geological structures for seismic risk studies: Bulletin of Seismological Society of America, 69, 135-158.

Angelier, J., Colletta, B., Chorowicz, J., Ortlieb, L., Rangin, C., 1981, Fault tectonics of the Baja California peninsula and the opening of the Sea of Cortez, Mexico: Journal of Structural Geology, 3, 347-357.

Atwater, T., 1970, Implications of plate tectonics for the Cenozoic tectonic evolution of western North America: Geological Society of America Bulletin, 81, 3513-3536.

Bender, B., 1983, Maximum likelihood estimation of $b$ values for magnitude grouped data: Bulletin of Seismological Society of America, 73, 831-851.

Boore, D.M., 1983, Stochastic simulation of high-frequency ground motions based on seismological models of the radiated spectra: Bulletin of Seismological Society of America, 73, 1865-1894.

Boore, D., Joyner, W.B., 1997, Site amplification for generic rock sites: Bulletin of Seismological Society of America, 87, 327-341.

Brune, J., 1970, Tectonic stress and the spectra of seismic shear wavesfrom earthquakes: Journal of Geophysical Research, 75, 4997-5009.

Busch, M.M., Arrowsmith, J.R., Umhoefer, P.J., Martínez- Gutiérrez, G., Toké, N.A., Brothers, D., DiMaggio, E.N., Maloney, S.J., Zielke, O., Buchanan, B., 2006, Late Quaternary faulting in the Cabo San Lucas-La Paz region, Baja California: Eos Transactions, American Geophysical Union, 87, abstract T41D-1612.

Busch, M.M., Coyan, J.A., Arrowsmith, J.R., Maloney, S.J., MartínezGutiérrez, G., Umhoefer, P.J., 2007, Late Quaternary faulting along the San Juan de los Planes fault zone, Baja California Sur, Mexico: Eos Transactions, American Geophysical Union, 88, abstract T41A-0357.

Comisión Federal de Electricidad (CFE), 1998, Manual de Diseño de Obras Civiles. Diseño por sismo, 193 p.; México D.F., Ed. Comisión Federal de Electricidad. Instituto de Investigaciones Eléctricas.

DeMets, C., 1995, A reappraisal of seafloor spreading lineations in the Gulf of California: Implications for the transfer of Baja California to the Pacific plate and estimates of Pacific-North America motion: Geophysical Research Letters, 22, 3545-3548.

Fletcher, J., Munguia, L., 2000, Active continental rifting in southern Baja California, Mexico: Implications for plate motion partitioning and the transition to seafloor spreading in the Gulf of California: Tectonics, 19, 1107-1123.

Fletcher, J.M., Pérez-Venzor, J.A., Gonzáles-Barba, G., Aranda-Gómez, J.J., 2003, Ridge-trench interactions and the ongoing capture of the Baja California microplate-New insights from the southern Gulf extensional province, in Geologic Transects across Cordilleran México (resumen), en Guidebook for Field Trips of the 99th Geological Society of America Cordilleran Section Annual Meeting,
2003: Puerto Vallarta, Jalisco, Universidad Nacional Autónoma de México, Instituto de Geología, 13-31.

Fletcher, J.M., Grove, M., Kimbrough, D., Lovera, O., Gehrels, G.E., 2007, Ridge-trench interactions and the Neogene tectonic evolution of the Magdalena shelf and southern Gulf of California: Insights from detrital zircon $\mathrm{U}-\mathrm{Pb}$ ages from the Magdalena fan and adjacent areas: Geological Society of America Bulletin, 119, 1313-1336.

Frankel, A., 1995, Mapping seismic hazard in the central and Eastern United States: Seismological Research Letters, 66, 8-21.

Hausback, B.P., 1984, Cenozoic Volcanic and Tectonic Evolution of Baja California Sur, Mexico: Berkeley, University of California, Ph.D. thesis, $72 \mathrm{p}$.

Lizarralde, D., Axen, G.J., Brown, H.E., Fletcher, J.M., GonzálezFernández, A., Harding, A.J., Holbrook, W.S., Kent, G.M., Paramo, P., Sutherland, F., Umhoefer, P.J., 2007, Variation in styles of rifting in the Gulf of California: Nature, 448, 466-469.

Maloney, S.J., Umhoefer, P.J., Arrowsmith, J.R., Martinez- Gutiérrez, G., Santillanez, A.U., Rittenour, T.R., 2007, Late Pleistocene-Holocene faulting history along the northern El Carrizal fault, Baja California Sur, Mexico, Earthquake recurrence at a persistently active rifted margin: Eos Transactions, American Geo-physical Union, 88, abstract T41A-0357.

Mayer, L., Vincent, K.R., 1999, Active tectonics of the Loreto area, Baja California Sur, Mexico: Geomorphology, 27, 243-255.

NGA, 2014, New Generation Attenuation Relationships for western US, revisado el 14 de Julio de 2014, disponible en http://peer.berkeley. edu/ngawest/index.html, Universidad de California en Berkeley.

Ortega, R., Gonzalez, M., 2007, Seismic-wave attenuation and source excitation in La Paz-Los Cabos, Baja California Sur, Mexico: Bulletin of Seismological Society of America 97, 545-556.

Ortega, R., Quintanar, L., 2010, Seismic evidence of a ridge-parallel strike-slip fault off the transform system in the Gulf of California: Geophysical Research Letters, 37.

Ortega, R., Quintanar, L., 2011, A comparison between P-wave and $\mathrm{S}$-wave propagation characteristics in the southern part of the Gulf of California, Mexico: Bulletin of Seismological Society of America, 101(3), $1270-1280$.

Oskin, M., Stock, J.M., Martín-Barajas, A., 2001, Rapid localization of Pacific-North America plate motion in the Gulf of California: Geology, 29, 459-462.

Plattner, C., Malservisi, R., Dixon,T.H., LaFemina, P., Sella, G.F., Fletcher, J., Suarez-Vidal, F., 2007, New constraints on relative motion between the Pacific plate and Baja California microplate (Mexico) from GPS measurements: Geophysical Journal International, 170, 1373-1380.

Stein, R., Toda, S., Parsons, T., Grunewald, E., 2006, A new probabilistic seismic hazard assessment for greater Tokyo: Philosophical Transactions of the Royal Society, 364, 1965-1968.

Stock, J.M., Hodges, K.V., 1989, Pre-Pliocene extension around the Gulf of California and the transfer of Baja California to the Pacific plate: Tectonics, 8, 99-115.

Stock, J.M., Lee, J., 1994, Do microplates in subduction zones leave a geological record?: Tectonics, 13, 1472-1488.

Tanner, J.G., Shepherd, J.B., 1997, Seismic hazard in Latin America and the Carribean, in Project Catalog ans Seismic Hazard Maps: Ottawa, International Development Research Center, 1, $143 \mathrm{p}$.

Umhoefer, P.J., Mayer, L., Dorsey, R.J., 2002, Evolution of the margin of the Gulf of California near Loreto, Baja California peninsula, Mexico: Geological Society of America Bulletin, 114, 849-868.

Umhoefer, P.J., Sutherland, F., Kent, G., Harding, A., Lizarralde, D., Schwennicke, T., Fletcher, J., Holbrook, W.S., Axen, G., 2008, Synchronous changes in rift-margin basins and initiation of the Alarcón spreading ridge and related transform fault, southwestern Gulf of California, in Geological Society of America: Houston, Texas, Geological Society of America Abstracts with Programs, 40, 151.

Weichert, D.H., 1980, Estimation of earthquakes recurence parameters for unusual observation periods for different magnitudes: Bulletin of Seismological Society of America, 70, 1337-1356. 
Wells, D.L., Coppersmith, K.J., 1994, New empirical relationships among magnitude, rupture length, rupture witdth, rupture area and surface displacement: Bulletin of Seismological Society of America, 8, $974-1009$.

Wesnousky, S. 1986, Earthquakes, quaternary faults, and seismic hazard in California: Journal of Geophysical Research, 91, 12587- 12631.

Manuscrito recibido: Julio 17, 2014

Manuscrito corregido recibido: Octubre 30, 2014

Manuscrito aceptado: Noviembre 2, 2014 